\title{
Wearable Internet of Things - from Human Activity Tracking to Clinical Integration
}

\author{
Poonam Kumari, Student Member, IEEE, Miguel López-Benítez, Senior Member, IEEE, Gyu Myoung \\ Lee, Senior Member, IEEE, Tae-Seong Kim, Atul S. Minhas, Member, IEEE
}

\begin{abstract}
Wearable devices for human activity tracking have been emerging rapidly. Most of them are capable of sending health statistics to smartphones, smartwatches or smart bands. However, they only provide the data for individual analysis and their data is not integrated into clinical practice. Leveraging on the Internet of Things (IoT), edge and cloud computing technologies, we propose an architecture which is capable of providing cloud based clinical services using human activity data. Such services could supplement the shortage of staff in primary healthcare centers thereby reducing the burden on healthcare service providers. The enormous amount of data created from such services could also be utilized for planning future therapies by studying recovery cycles of existing patients. We provide a prototype based on our architecture and discuss its salient features. We also provide use cases of our system in personalized and home based healthcare services. We propose an International Telecommunication Union based standardization (ITU-T) for our design and discuss future directions in wearable IoT.
\end{abstract}

\section{INTRODUCTION}

Internet of Things (IoT) has emerged as a disruptive technology finding its applications in smart cities, retail, agriculture, industrial automation, electronic-Health (e-health), mobile-Health (m-health), and many others [1-2]. Using the IoT, we can create application-specific solutions by interconnecting physical objects through internet and allow them to collaborate with each other to achieve assigned tasks. Interconnected wearable devices for physiological activity tracking are rapidly emerging and forming a new segment-"Wearable IoT (WIoT)" [3]. Most commonly used wearable sensors include wearable body temperature sensors, pulse and blood oxygen level sensors, accelerometers for motion sensing, airflow sensors, electrocardiograms, and galvanic skin response sensors [4]. Out of these, motion sensors have been most widely used in commercial products with a wide range of applications [3-9].

Future developments in WIoT will influence the way personalized health and wellness is being managed until today. One of the focus of IoT research in healthcare is to

Poonam Kumari and Tae-Seong Kim are with the Department of Biomedical Engineering, Kyung Hee University, Yongin-si, South Korea (e-mail: chauhan.poonam5@gmail.com, tskim@khu.ac.kr).

Miguel López-Benítez is with the Department of Electrical Engineering and Electronics, University of Liverpool, Liverpool, UK (e-mail: M.Lopez-Benitez@liverpool.ac.uk).

Atul S Minhas is with the Centre for Pre-clinical Imaging, Department of Cellular and Molecular Physiology, University of Liverpool, UK (e-mail: atul.minhas@liverpool.ac.uk).

Gyu Myoung Lee is with the Department of Computer Science, Liverpool John Moores University, Liverpool, UK (e-mail: g.m.lee@ljmu.ac.uk). transform the community healthcare using real-time information to support self-management of health and wellbeing. This can reduce burden on primary health centers and facilitate timely interventions in emergency situations [2]. Self-management can also reduce burden on the rehabilitation centers responsible for post-surgery patient recovery by managing and monitoring minor rehabilitation activities at home and relying on rehabilitation centers only for designated purposes. The physiological activity tracking data generated at home can also be used to improve the existing therapies and study recovery variations over a wider population. Motion sensors combined with temperature and pulse sensors will see a wider acceptance in public and clinical practice $[2,10]$.

With the advent of Micro Electromechanical Systems (MEMS) technology, the motion detecting sensors have advanced from primitive costly and bulky sensors to existing miniature low cost sensors [11]. This allowed them to be integrated into smartphones and wearables like smart watches and activity tracking devices. Sensors with multiple features and capabilities are now available which can provide real time raw data of acceleration, angular motion, and magnetic field based on Earth's gravity. Further processing of these data can provide services like activity charts over a span of time for steps, walking pattern, sleeping pattern, and location information. Users can also get notifications about their achieved activity goals or reminders if they could not achieve their set goal. With the emergence of cloud computing, users now have the option to use application programming interfaces (APIs) to integrate their activity data to cloud servers for advanced analytics [4].

The current activity tracking devices have proven highly beneficial for individual use. However, they lack data integrity, interoperability, and standardization. Currently no industry standard APIs exist to span all devices. Some studies have also shown differences in the data acquired from devices of different vendors [4]. ITU-T has developed various standards related to healthcare services [12]. However, governments and commercial product companies have been lagging behind in implementing these standards. Due to the absence of reliability and lack of standardization, the data acquired from human activity tracking devices cannot be used for clinical purpose. Some studies have proposed utilizing the smartphone based accelerometers for activity monitoring [7]. However, such devices become cumbersome and sometimes give inaccurate results due to the larger size of smartphones.

In this paper, we propose an edge computing based architecture for WIoT for personalized and home based healthcare services. We provide an example of prototyping this system and the standardization necessary to translate this architecture to clinical practice. We also discuss the upscaling 
of this architecture from home based services to community services in a built environment like rehabilitation center.

\section{ARCHITECTURE OF WEARABLE IOT FOR HOME BASED HEALTHCARE SERVICES}

Our complete architecture for home based healthcare services is elaborated in following sections.

\section{A. Wearable Human Activity Tracking Device}

Fig 1 shows the wearable human activity tracking device. It consists of activity tracking MEMS sensors such as 3-axis accelerometer, 3-axis gyroscope and 3-axis magnetometer. Sensors collect data from human body which is then processed by an onboard Digital Motion Processor (DMP) and stored in data registers. The microcontroller unit (MCU) fetches data from the data registers of the activity tracking device using Inter-Integrated Circuit (I2C) or Serial Peripheral Interface (SPI) communication protocol. The data is then sent to edge or cloud using short-range communication protocols such as IEEE 802.11 (WiFi) [12]. Another memory connected to the microcontroller is sometimes used in case there is a failure in communicating with edge. The device also has an inbuilt temperature sensor to sense the heating in electronics and shutdown the system in case of excessive heating. The device is powered by $5 \mathrm{~V}$ battery. Multiple activity tracking devices can be used in a household either by a single person or by multiple people occupying a single premises. Each device has its own unique identification name and security keys. This allows them to either communicate with each other independently or communicate with an edge computing device located in indoor premises (Fig 2).

\section{B. Edge Computing Device}

Fig 2 shows an edge computing device (ECD) which has memory for data storage, and communicates information between wearable devices and cloud. The ECD configures the wearable devices to operate in two modes, viz. direct device to device communication, or device to ECD communication. Excessive data from wearable devices is stored in edge memory and cleaned for pre-processing. The edge computing device runs on a light operating system such as Android or Raspbian [13]. It also has the capability to provide analytics if necessary. However, edge computing algorithms are mostly "If This Then That" (IFTTT) based web-services [14]. The edge acts as an interface between the activity tracking device and cloud and has bidirectional communications with both. Keeping the edge in indoor premises enhances data protection and security by allowing users to choose from various security options available in market. Besides, a user gets full control over the data management and storage.

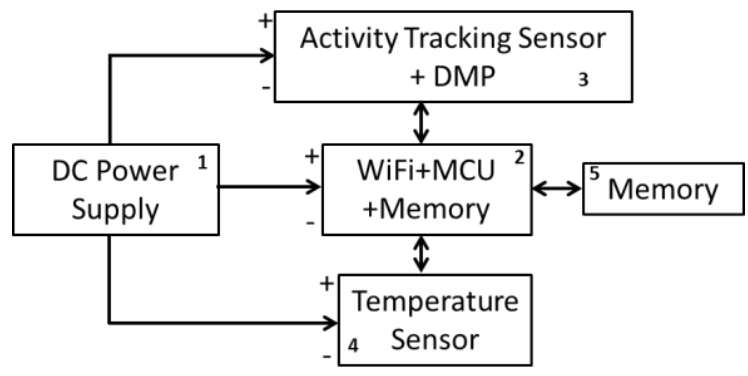

Figure 1. Wearable activity tracking device

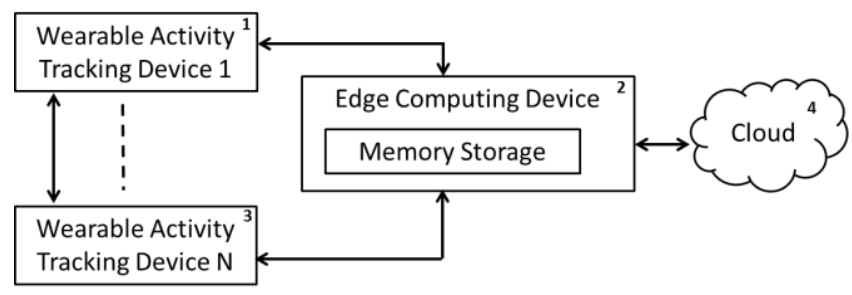

Figure 2. Wearable IoT system with $\mathrm{N}$ tracking devices and edge and cloud sharing the computing and storage

\section{Cloud Computing and Analytics Service}

Fig 2 shows a cloud which receives data from ECD and runs advanced analytics. The transfer of data from edge to cloud is done with the permission of user. However, user has no control on cloud data analytics and management. All the cloud based artificial intelligence algorithms for activity tracking needs to be approved and standardized by healthcare regulatory bodies. Cloud service providers need to get approvals from the federal agencies of respective countries for the safety and reliability of outcomes of their algorithms as they are used for human health management.

\section{Clinical Integration}

Analytics provided by cloud are used for clinical decision making. The role of cloud in the decision making is twofold: to provide initial screening services which would normally be provided by nurses or doctors in dispensaries, and to take decisions for further examination by doctors. The clinical integration of calculated analytics needs to be done in consultation with healthcare researchers and clinical practitioners. The analytics need to form an integral part of the hospital management software and included in the patient specific reports such as electronic health records (EHR) and electronic medical records (EMR). A proper training to hospital staff is necessary to understand the analytics and provide feedback to the patients.

\section{STANDARDIZATION}

Our WIoT system recommends IUT-T standards for e-health monitoring services. ITU-T Recommendation Y.2065 ("Service and capability requirements for e-health monitoring services") describes the service requirements for the support of e-health monitoring services, and it specifies the corresponding capability requirements. ITU-T Recommendation Y.2075 ("Capability framework for e-health monitoring services") specifies the capability framework for support of the requirements of e-health monitoring (EHM) services. Recommendation ITU-T H.860 ("Multimedia e-health data exchange services: Data schema and supporting services") specifies a common health schema applicable to a wide range of health systems - e.g., clinical and wellness and describes the supporting services and systems architecture for a health data exchange that allows an exchange of multimedia health data between a health provider, a controlling function and a patient. The services this Recommendation describes include both point-of-care and personal healthcare operating systems.

The draft Recommendation Y.IoT-EH-PHE ("Performance evaluation frameworks of e-health systems in the IoT") specifies performance evaluation frameworks of 
e-health systems in the IoT for e-health services. From information and communication technologies point of view, e-health services are classified. Performance evaluation factors applicable for e-health systems in the IoT are specified.

\section{PERSONALIZED AND HOME BASED HEALTHCARE SERVICES}

Chronic diseases such as diabetes, obesity, and cardiovascular disorders affect the lives of millions of people around the word. Current clinical practice to treat such patients is through consultation, prescription medicines, and regular monitoring by hospital visits. The treatment protocol varies from person to person and country to country. Using our proposed system, we can collect data about the daily activities of such patients in real-time. At home premises, edge computing based services like alarm, text message, or phone call could be triggered in case the prescribed activities are not performed by patients. The edge computer can consult with cloud and take a decision of recommending patient for personal examination by health consultant if necessary.

Post-surgery physiotherapy is a necessary part of rehabilitation program for treatment and management of musculoskeletal disorders [14-15]. However, most European countries have a long waiting list at their national healthcare service providers. Using our proposed architecture, a home based rehabilitation program can be established. Initial guidelines could be provided by healthcare service provider after which edge computer will take charge and monitor the activity of patient in home. As an example, edge computer can keep a track of whether patient moved his limbs within the prescribed limit, or whether patient's daily mobility was within prescribed limit. Such data can be shared with cloud for analysis and feedback by healthcare consultants. Besides, advanced data analytics applied on data could provide new insights into the course of rehabilitation.

\section{PROTOTYPING}

Wearable device is meant to be worn on body. This puts the small size as the major design constraint. Considering this, we chose MPU9250 (MS) as the 9-axis motion sensing device and Particle Photon (PP) as the microcontroller [16-17]. Their actual dimensions are shown in Fig $3 b$. The PP is a miniature sized development board with Broadcom microcontroller with WiFi capability [18]. In actual assembly, we mounted MS over PP and connected a 5V battery to PP. The MS takes the power supply from the $3.3 \mathrm{~V}$ pin of PP. Various other pin connections are shown in Fig 3c. For edge computing, we used Raspberry Pi 3 (RPi) board which is a low cost computer running Raspbian operating system. It has quad-core 64-bit ARM Cortex (1.2 GHz) processor, 1GB SDRAM, and integrated 802.11n wireless LAN module.

We setup the PP using WiFi network and connected it with an Android based smartphone application provided by Particle Photon. We also developed a smartphone application which acted as a user interface to send instructions to PP about when to start/stop acquiring data from MS. Besides, the smartphone application also had the capability to control the data communication between PP and RPi. We programmed PP using the Arduino based application programming environment provided by Particle [16]. The RPi programs were based on python scripts.

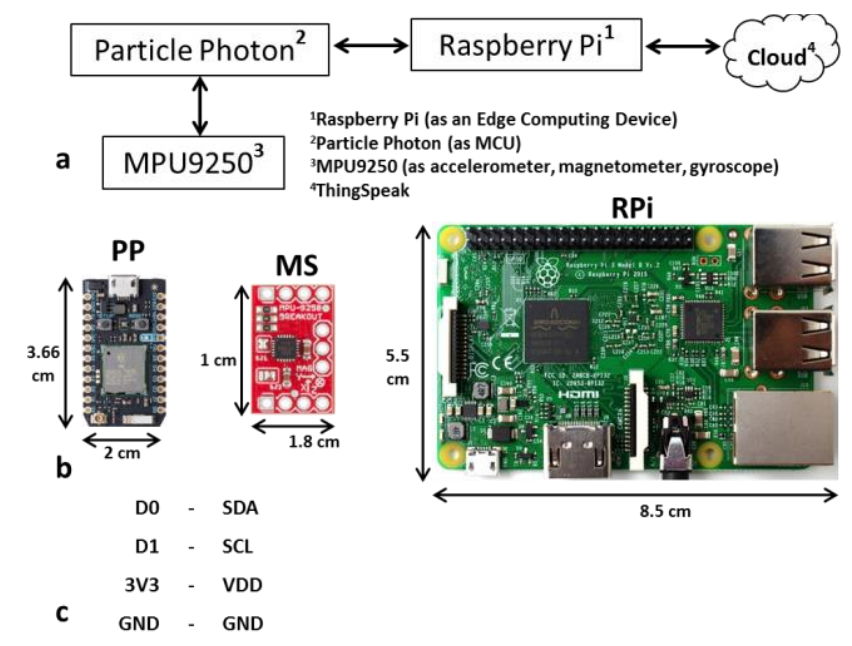

Figure 3. Prototype of our Wearable IoT system. The block diagram with interconnections is shown in (a), the dimensions of major components used is shown in (b), and pin connections between Particle Photon and MPU9250 are shown in (c)

For cloud based analytics, we used ThingSpeak cloud platform [19]. Using PP's programming platform, we created a webhook to cloud ThingSpeak server. This enabled us to send data to a channel in ThingSpeak. After the data reached at an assigned channel, we performed web-analytics to analyze and visualize the data. We can also interact with social media, web services, and other devices. We also ran a comparative study of checking the latency in data transfer from Photon to its own cloud and to the ThingSpeak cloud. We found that in ThingSpeak cloud server, data samples were updated every 20s, but in Particle's self-managed server, the data transfer was in real-time. We also ran tests with serial communication using USB and found that serial communication gave a data sample update every $2 \mathrm{~s}$.

\section{DISCUSSION}

The cloud service is managed by a third party and the data transfer rates are slow for real-time applications. So, cloud cannot be used for fast computations and must be only used for retrospective analysis of patient data. In case data sampling in milli-seconds or more is needed, we propose using the edge computer with WiFi connectivity. It is worth noticing that we are using a freely available cloud service and data sampling could be increased by choosing a paid cloud service such as Google Cloud, Microsoft Azure, or Amazon Web Services. We used webhook for transferring data to a freely available cloud space, but one can also use other web services like Node.js and HTTP to create webservers and transfer data for storage and retrospective analysis.

We have used I2C communication in our prototype which is slower compared to serial communication. Care must be taken not to use the connecting cables very long as this may create data losses or disconnections. We used WiFi as a communication medium within household premises. However if the goal is to create a simple human activity tracking application such as onset/offset counting of activities, the simple and cost-effective protocols such as Zigbee could be used to transfer data to edge computer. 
We used RPi as an edge computing device. However, in a large household such as bungalow, workplaces, or multi-story buildings, we can deploy either multiple RPis or use a powerful workstation to manage the communication. Under such circumstances, the building management will be responsible for managing the edge computer.

We proposed the architecture for home based healthcare service, but within the framework of IoT, this architecture can be easily extended to a community healthcare service provider. In this upscaling, multiple activity monitoring devices will be used by multiple patients and they all will send their data to a centrally located server. An efficient design of such use case is to assign a cluster of activity trackers to a particular RPi and multiple such RPis communicate with each other and with the centralized server. Such architecture could be suitable for community rehabilitation centers.

In some use case, the artificial intelligence algorithms in our design may run in edge computer. Care must be taken not to write memory in-efficient programs to implement the algorithms as there is shortage of memory in edge and extra memory may make the edge a costly solution. We may rely on FPGA or GPU programming for such tasks.

\section{CONCLUSION}

We proposed a WIoT architecture for activity tracking in household premises. Our design is robust to data security, and integrates cloud with edge for efficient clinical services. Commercial systems based on such an architecture are not yet available but will come soon in near future. Our design proposes to follow the ITU-T standardization guidelines and expects the government bodies to make policies for their implementation. The architecture is designed considering users in mind and hence ease of use, security and cost-effectiveness are given priority. Considering its cost-effectiveness, the design could also be used to provide efficient healthcare services to the people of low and medium income countries (LMICs). Such systems could be highly beneficial for LMICs as they have burgeoning population but shortage of staff.

\section{REFERENCES}

[1] $\mathrm{S} \mathrm{Li}$, and L D Xu, "The internet of things: a survey," Information Systems Frontiers, vol. 17, pp. 243-259, Apr 2015.

[2] D V Dimitrov, "Medical Internet of Things and big data in healthcare," Healthcare Information Research, vol. 22, pp. 156-163, Jul 2016.

[3] S Hiremath, G Yang, and K Mankodiya, "Wearable Internet of Things: concept, architectural components and promises for person-centered healthcare," in 4th Intl. Conf. on Wireless Mobile Communication and Healthcare, pp. 304-307, Nov 2014.

[4] T Davenport, and J Lucker, "Running on data: activity trackers and the Internet of Things," in Deloitte Review, issue 16, Jan 2015.

[5] M Zubair, K Song, and C Yoon, "Human activity recognition using wearable accelerometer sensors," in 2016 IEEE Int. Conf. on Consumer Electronics-Asia (ICCE-Asia), pp. 1-5, Oct 2016.

[6] F Attal, S Mohammed, M Dedabrishvili, F Chamroukhi, L Oukhellou, and Yacine Amirat, "Physical human activity recognition using wearable sensors," in Sensors, vol. 15, pp. 31314-31338, Dec 2015.

[7] A Bayat, M Pomplun, and Duc A. T, "A study on human activity recognition using accelerometer data from smartphones," 11th Int.
Conf. on Mobile Systems and Pervasive Computing, pp. 450-457, vol. 34, 2014

[8] P Bonato, "Wearable sensors/systems and their impact on biomedical engineering," IEEE Engineering in Medicine and Biology Magazine, pp. 18-20, Jun 2003.

[9] H Amroun, N Quarti, and M Ammi, "Recognition of human activity using Internet of Things in a non-controlled environment," ICARCV, Nov 2016.

[10] A Doherty, D Jackson, Nils Hammerla, T Plotz, P Olivier, et.al. "Large Scale Population Assessment of Physical Activity Using Wrist Worn Accelerometers: The UK Biobank Study," Plos One, vol. 12, pp. 1-14, Feb 2017.

[11] P L Walter, "The History of the Accelerometer 1920s-1996 - Prologue and Epilogue, 2006," in sound and Vibration Magazine, pp. 84-92, Jan 2007.

[12] Laura DeNardis, "E-health Standards and Interoperability," in ITU-T Technology Watch Report, Apr 2012.

[13] http://www.raspberrypi.org

[14] http://https://ifttt.com/

[15] https://www.rnoh.nhs.uk/our-services/rehabilitation-guidelines

[16] H Zhou, and $\mathrm{H} \mathrm{Hu}$, "Human motion tracking for rehabilitation-A survey," Biomedical Signal Processing and Control, vol. 3, pp. 1-18, Jan 2008.

[17] https://www.invensense.com/products/motion-tracking/9-axis/mpu-9 $250 /$

[18] https://www.particle.io/

[19] https://thingspeak.com/ 